NOTICE: This is the author's version of a work that was accepted for publication in Signal Processing. Changes resulting from the publishing process, such as peer review, editing, corrections, structural formatting, and other quality control mechanisms may not be reflected in this document. Changes may have been made to this work since it was submitted for publication. A definitive version was subsequently published in Signal Processing [90, 12, 2010] DOI 10.1016/j.sigpro.2010.05.018 


\title{
A Dual Parametrization Approach to Nyquist Filter Design
}

\author{
C.Z. Wu and K.L. Teo, Senior Member
}

\begin{abstract}
In this paper, the optimum design of a factorable Nyquist filter with the intersymbol interference (ISI) being exactly zero is formulated as a nonlinear optimization problem with continuous inequality constraints. A computational strategy based on iterative scheme combined with an improved dual parametrization method is devised. Trade-off between robustness to timing jitter and small stopband attenuation is achieved via an adjustment of a parameter. Some examples are solved using the proposed method.
\end{abstract}

\section{Index Terms}

Nyquist filter, timing jitter, exact intersymbol interference, optimum design, computational strategy

\section{INTRODUCTION}

The impulse response of a Nyquist filter (also referred to as Mth band filter) is required to be exactly zero at the Nyquist rate, except for one point. This is to ensure that the intersymbol interference (ISI) is exactly zero. It has many applications in digital signal processing, such as filter banks, nonuniform sampling, interpolation filters. As a consequence, the design of such a filter has been extensively studied in the past two decades [1-10].

In the design of a Nyquist filter, it is required to find a set of coefficients such that the corresponding Nyquist filter will achieve the following conditions. (i) the zero ISI condition is satisfied; (ii) it is robust to timing jitter; and (iii) it produces small energy in the stopband. In [2], a method based on the periodical nonuniform sampling theory is developed for the design of this filter. The robustness to timing jitter is achieved through maximizing the energy in the middle of the impulse response. However, it does not address the nonnegativity requirement of the frequency response. This requirement is essential for achieving a spectral factorable sequence. In [3], the windowing method combined with the Remez exchange method is used for the design of this filter, where the robustness to

This paper is partially supported by a research grant from the Australian Research Council, National Natural Science Foundation of China (10826096), Chongqing Municipal Education Commission (KJ090802).

C.Z. Wu is with the School of Mathematics and Computer Science, Chongqing Normal University, Chongqing, China and the Department of Mathematics and Statistics, Curtin University of Technology, Perth, WA, Australia. Email address: changzhiwu@yahoo.com, Phone: 61-8-92663144, Fax: 61-8-92663197.

K.L. Teo is with the Department of Mathematics and Statistics, Curtin University of Technology, Perth, WA, Australia. Email address: k.1.teo@curtin.edu.au. Phone: +61-8-9266-1115, Fax: +61-8-9266-3197. 
timing jitter is achieved by the windowing method. However, the exactly zero crossings cannot be maintained in the implementation of the Remez exchange algorithm. In [4], the design of a square-root Nyquist filter is considered. The zero crossings and the robustness to timing jitter are determined based on the ideal target impulse response and some penalty parameters. However, there is no systematic method that can be used to choose the ideal target such that the designed filter is robust to timing jitter. In [5], the design of a factorable FIR Nyquist filter is formulated as a quadratic optimization problem with continuous linear inequality constraints. However, there is no consideration on the issue relating to the robustness to timing jitter.

In this paper, we consider the design of a factorable Nyquist filter with reference to the three criteria mentioned above. The exact zero-crossings are achieved by pre-fixing the corresponding filter coefficients to be zero. The robustness to timing jitter is obtained through minimizing the tail energy of the impulse response while maintaining the total energy of the impulse response as being a constant. Thus, the optimal design of such a filter can be formulated as a nonlinear optimization problem with continuous inequality constraints. An iterative scheme is developed to solve this optimization problem. In each iteration, a quadratic semi-infinite optimization problem with linear inequality constraints is required to be solved. There are many methods available to solve this type of semi-infinite optimization problem. Examples include the constraint transcription constraints method [11], the discritization method [12]. Here, we will develop an improved dual parametrization method based on [14] and [13] to solve this optimization problem. This approach is much superior to existing methods, such as those reported in [11], [12] and [15].

\section{Problem Formulation}

Consider the following FIR filter, where the number $N$ of the length of the filter is odd.

$$
H(z)=\sum_{n=0}^{N-1} h_{n} z^{-n} .
$$

If $H(z)$ is an $M$ th band filter, then $\left\{h_{n}\right\}_{n=0}^{N-1}$ satisfies the following conditions:

1) $H(z)$ is a symmetric filter, i.e., $h_{L+k}=h_{L-k}, k=0,1, \cdots, L$, where $L=(N-1) / 2$;

2) Nyquist criterion, i.e.

$$
h_{n}= \begin{cases}1, & n=L \\ 0, & n=L+k M, k= \pm 1, \cdots, \pm\lfloor L / M\rfloor, \\ \text { arbitrary, } & \text { otherwise }\end{cases}
$$

Suppose that the passband and stopband of the $M$ th filter are $\left[0, w_{p}\right]$ and $\left[w_{s}, \pi\right]$, respectively. Then, $w_{p}$ and $w_{s}$ are given by

$$
w_{p}=\frac{(1-\beta) \pi}{M}, w_{s}=\frac{(1+\beta) \pi}{M}
$$


where $\beta, 0 \leq \beta \leq 1$, is the roll-off factor. Furthermore, both the passband magnitude and phase error are decided by the stopband error [6]. Let $H\left(e^{j w}\right)=H(w) e^{-j L w}$. Then,

$$
H(w)=1+\sum_{k=1}^{L} 2 h_{L-k} \cos (k w)=1+\boldsymbol{x}^{T} \boldsymbol{C}(w),
$$

where $\boldsymbol{x}=\left[2 h_{L-1}, \cdots, 2 h_{0}\right]^{T}, \boldsymbol{C}(w)=[\cos w, \cdots, \cos (L w)]^{T}$. The stopband energy of this filter is

$$
E_{s}(\boldsymbol{x})=\int_{w_{s}}^{\pi}|H(w)|^{2} d w=\boldsymbol{x}^{T} T \boldsymbol{x}+2 \boldsymbol{p}^{T} x+1,
$$

where $T=\left(t_{k, l}\right)_{L \times L}$ is an $L \times L$ matrix with its elements $t_{k, l}, k, l=0,1, \cdots, L-1$, given by

$$
t_{k, l}= \begin{cases}1, & \text { if } k=0, l=0, \\ \pi-w_{s}-\sin \left((k+l) w_{s}\right) /(2(k+l)), & \text { if } k=l, \\ -\sin \left((k+l) w_{s}\right) /(2(k+l)) & \text { if } k \neq l, \\ -\sin \left((k-l) w_{s}\right) /(2(k-l)), & \end{cases}
$$

while $\boldsymbol{p}=\left[p_{0}, p_{1}, \cdots, p_{L-1}\right]^{T}$ with $p_{k}=-\sin \left(k w_{s}\right) / k, k=0,1, \cdots, L-1$. Since $H(z)$ is an $M$ th band filter and $\boldsymbol{x}$ is determined by its coefficients, we have $x_{k M}=0$. We delete those zero elements in $\boldsymbol{x}$ and let the remanning elements be denoted collectively as $\tilde{\boldsymbol{x}}$ which has $L-\lfloor L / M\rfloor+1$ elements, where $\lfloor\cdot\rfloor$ is the floor function. Let $\tilde{L}=L-\lfloor L / M\rfloor+1$, and let $\tilde{T}, \tilde{\boldsymbol{C}}(w)$ and $\tilde{\boldsymbol{p}}$ be the corresponding reduced versions of $T, \boldsymbol{C}(w)$ and $\boldsymbol{p}$, respectively. Then, for an $M$ th band filter, (3) and (4) can be re-written as

$$
\begin{aligned}
& H(w)=1+\tilde{\boldsymbol{x}}^{T} \tilde{\boldsymbol{C}}(w), \\
& E_{s}(\tilde{\boldsymbol{x}})=\tilde{\boldsymbol{x}}^{T} \tilde{T} \tilde{\boldsymbol{x}}+2 \tilde{\boldsymbol{p}}^{T} \tilde{\boldsymbol{x}}+1,
\end{aligned}
$$

respectively.

Let

$$
E_{\text {tail }}=\sum_{k=M k}^{\tilde{L}} \tilde{x}_{k}^{2}, E_{\text {total }}=1+\tilde{\boldsymbol{x}}^{T} \tilde{\boldsymbol{x}}
$$

where $\tilde{x}_{k}$ is the $k$ th element of $\tilde{\boldsymbol{x}}, E_{\text {tail }}$ is the tail energy and $E_{\text {total }}$ is the total energy of the impulse response of $H(z)$. From [1] and [2], we see that the effect of timing jitter of this Nyquist filter can be reduced through maximizing the main lobe energy or minimizing the tail energy of the impulse response subject to maintaining the total energy being a constant. Since we do not know the value of the total energy in advance, the reduction of the timing jitter effect is to be addressed by minimizing the following function

$$
E_{0}(\tilde{\boldsymbol{x}})=\frac{\sum_{k=M}^{\tilde{L}} \tilde{x}_{k}^{2}}{1+\tilde{\boldsymbol{x}}^{T} \tilde{\boldsymbol{x}}}=\frac{\tilde{\boldsymbol{x}}^{T} R \tilde{\boldsymbol{x}}}{E_{\text {total }}}
$$

where

$$
R=\left[\begin{array}{cc}
0_{M \times M} & \\
& I_{(\tilde{L}-M+1) \times(\tilde{L}-M+1)}
\end{array}\right] .
$$


Now, the optimal design of an $M$ th band filter such that it is robust to timing jitter can be formulated as the following optimization problem

Problem P1

$$
\min _{\tilde{\boldsymbol{x}}} J(\tilde{\boldsymbol{x}})=\tilde{\boldsymbol{x}}^{T} \tilde{T} \tilde{\boldsymbol{x}}+\lambda \frac{\tilde{\boldsymbol{x}}^{T} R \tilde{\boldsymbol{x}}}{E_{\text {total }}}+2 \tilde{\boldsymbol{p}}^{T} \tilde{\boldsymbol{x}}+1,
$$

subject to the continuous linear inequality constraints

$$
\begin{aligned}
& H(w)=1+\tilde{\boldsymbol{x}}^{T} \tilde{\boldsymbol{C}}(w) \geq 0, \text { for all } w \in\left[w_{s}, \pi\right] \\
& H(w)=1+\tilde{\boldsymbol{x}}^{T} \tilde{\boldsymbol{C}}(w) \leq \delta(w), \text { for all } w \in\left[w_{s}, \pi\right]
\end{aligned}
$$

where $\lambda$ is a weighting factor.

Remark 1: (10) is to ensure that the obtained Nyquist filter $H(z)$ is factorable. This is required, as in many applications, such as in filterbanks [16], the Nyqusit filter is required to be factorable. (11) is the constraint on the peak-sidelobe level, where $\delta(w)$ is the maximum allowable height of the sidelobe at each frequency in the stopband.

\section{DESIGN PROCEDURE}

In Problem P1, we are seeking to optimize $J(\tilde{\boldsymbol{x}})$ with respect to $\tilde{\boldsymbol{x}}$. However, $E_{\text {total }}$ is not available at each frequency in the stopband in the current iteration. In our design procedure, we estimate $E_{\text {total }}$ by using $\tilde{\boldsymbol{x}}$ obtained in the previous iteration. Our numerical experience shows that this design strategy is effective. The iterative procedure can now be stated as follows. Let $\tilde{\boldsymbol{x}}^{(k)}$ denote the $\tilde{\boldsymbol{x}}$ obtained in the $k$ th iteration. Then

$$
\begin{aligned}
& J_{k}\left(\tilde{\boldsymbol{x}}^{(k)}\right)=\left(\tilde{\boldsymbol{x}}^{(k)}\right)^{T} \tilde{T} \tilde{\boldsymbol{x}}^{(k)} \\
& +\frac{\lambda}{\left(\tilde{\boldsymbol{x}}^{(k-1)}\right)^{T} \tilde{\boldsymbol{x}}^{(k-1)}}\left(\tilde{\boldsymbol{x}}^{(k)}\right)^{T} R \tilde{\boldsymbol{x}}^{(k)}+2 \tilde{\boldsymbol{p}}^{T} \tilde{\boldsymbol{x}}^{(k)}+1
\end{aligned}
$$

Since $\tilde{\boldsymbol{x}}^{(k-1)}$ is known in the $k$ th iteration, the cost function $J_{k}\left(\tilde{\boldsymbol{x}}^{(k)}\right)$ is in a quadratic form in terms of $\tilde{\boldsymbol{x}}^{(k)}$. Thus, in the $k$ th iteration, we only need to solve the following linear quadratic semi-infinite programming problem.

Problem P2

$$
\min _{\tilde{\boldsymbol{x}}} J(\tilde{\boldsymbol{x}})=\tilde{\boldsymbol{x}}^{T} Q \tilde{\boldsymbol{x}}+2 \tilde{\boldsymbol{p}}^{T} \tilde{\boldsymbol{x}}+1
$$

subject to the constraints

$$
\begin{aligned}
-\tilde{\boldsymbol{x}}^{T} \tilde{\boldsymbol{C}}(w) & \leq 1, \text { for all } w \in\left[w_{s}, \pi\right] \\
\tilde{\boldsymbol{x}}^{T} \tilde{\boldsymbol{C}}(w) & \leq \delta(w)-1, \text { for all } w \in\left[w_{s}, \pi\right]
\end{aligned}
$$

where $\tilde{\lambda}=\lambda /\left(\tilde{\boldsymbol{x}}^{(k-1)}\right)^{T} \tilde{\boldsymbol{x}}^{(k-1)}$, and $Q=\tilde{T}+\tilde{\lambda} R$ is a symmetric positive definite matrix.

Problem P2 is a linear quadratic semi-infinite optimization problem. There are many numerical methods available for solving this class of problems, such as the constraint transcription method (see [11]), the discritization method, 
(see [12]), and the dual parametrization techniques (see [13] and [14]). In this paper, we combine the methods in [13] and [14], forming an improved method. Let us introduce the Dorn's dual of Problem P2 given below.

Problem DP:

$$
\begin{aligned}
& \min _{\tilde{\boldsymbol{x}}, \boldsymbol{v}} \tilde{\boldsymbol{x}}^{T} Q \tilde{\boldsymbol{x}}+\int_{w_{s}}^{\pi}[1, \delta(w)-1] d\left[v_{1}(w), v_{2}(w)\right]^{T}, \\
& \text { s.t. } 2 Q \tilde{\boldsymbol{x}}+\mathbf{2} \tilde{\boldsymbol{p}}^{\boldsymbol{T}}+\int_{w_{s}}^{\pi} \tilde{\boldsymbol{C}}(w) d\left(v_{2}(w)-v_{1}(w)\right)=0 \\
& \boldsymbol{v}=\left[\begin{array}{c}
v_{1} \\
v_{2}
\end{array}\right] \in M^{+}\left(\left[w_{s}, \pi\right]\right), \tilde{\boldsymbol{x}} \in \mathbb{R}^{\tilde{L}+1},
\end{aligned}
$$

where $M^{+}\left(\left[w_{s}, \pi\right]\right)$ denotes the set of nonnegative bounded regular Borel measures on $\left[w_{s}, \pi\right], \boldsymbol{v}=\left[v_{1}, v_{2}\right]^{T}$.

A discretized version of Problem DP with $k$ active constraints is defined as follows.

Problem $D P_{k}$

$$
\begin{aligned}
& \min _{\tilde{\boldsymbol{x}}, \boldsymbol{\mu}, \boldsymbol{w}} \tilde{\boldsymbol{x}}^{T} Q \tilde{\boldsymbol{x}}+\sum_{i=1}^{k}\left(\mu_{i, 1}+\mu_{i, 2}\left(\delta\left(w_{i}\right)-1\right)\right), \\
& \text { s.t. } 2 Q \tilde{\boldsymbol{x}}++\mathbf{2} \tilde{\boldsymbol{p}}^{\boldsymbol{T}}+\sum_{i=1}^{k} \tilde{\boldsymbol{C}}\left(w_{i}\right)\left(\mu_{i, 2}-\mu_{i, 1}\right)=0 \\
& \boldsymbol{\mu}_{i}=\left[\begin{array}{l}
\mu_{i, 1} \\
\mu_{i, 2}
\end{array}\right] \geq 0, i=1, \cdots, k .
\end{aligned}
$$

Let $\boldsymbol{\mu}=\left(\mu_{i, 1}, \mu_{i, 2}, \cdots, \mu_{k, 1}, \mu_{k, 2}\right)$ and $\boldsymbol{w}=\left(w_{1}, w_{2}, \cdots, w_{k}\right)$. It has been shown [13] that if $\tilde{\boldsymbol{x}}^{*}$ is the optimal solution of Problem P2, then there exists a measure $\boldsymbol{v}^{*}$ which has finite support of no more than $\tilde{L}+1$ points such that $\left(\tilde{\boldsymbol{x}}^{*}, \boldsymbol{v}^{*}\right)$ is an optimal solution of Problem DP. Based on this, an iterative procedure is proposed. In each iteration, the points which are violating the constraints (14) and (15) with respect to the current $\tilde{\boldsymbol{x}}$ are adaptively chosen from $\left[w_{s}, \pi\right]$. Let these points be referred to collectively as $\boldsymbol{w}$. Then, Problem $D P_{k}$ with such an $\boldsymbol{w}$ is just a quadratic optimization problem with $\tilde{L}$ equality constraints (i.e., constraints (17)). It can be solved easily. This process is repeated. However, the number of elements in $\boldsymbol{w}$ may become very large after some iterations. To overcome this, a novel method is developed in [14], where in each iteration, the number of the active points is restricted to be $\tilde{L}+2$. However, this method is not efficient for cases when the number of the required active points is far smaller than the dimension of the decision vector to be minimized. In this paper, these two methods are combined, forming an improved method for solving Problem P2. First, we use the adaptive scheme in [13] to solve Problem $D P_{k}$. When the number of active points is increased to $\tilde{L}+3$, we then change over to use the method in [14] to choose $\tilde{L}+2$ points from these $\tilde{L}+3$ active points such that the optimal solution $\tilde{\boldsymbol{x}}$ of Problem $D P_{k}$ with $k=\tilde{L}+2$ is the same as that of Problem $D P_{k}$ with $k=\tilde{L}+3$. This procedure is stated as follows.

\section{Algorithm 1:}

Step 1 Set $\tilde{\boldsymbol{x}}^{(0)}$ as an initial point, $\varepsilon>0$ as a small number, $\Delta_{i}=\left\{w_{i, j} \in\left[w_{s}, \pi\right]: j=1, \cdots, k_{i}\right\}, i=1, \cdots$, as a 
sequence of parametrization sets, satisfying

$$
\max _{w \in\left[w_{s}, \pi\right]} \min _{w^{\prime} \in \Delta_{i}}\left|w-w^{\prime}\right| \rightarrow 0 \text { as } i \rightarrow \infty
$$

Step 2 Let $E^{0}=\phi$. Set $i=0$.

Step 3 Set $i=i+1$. If the number of points in $V_{i}$ is less than $\tilde{L}+3$, go to Step 4. Otherwise, let $E_{i}=V_{i}$ and go to Step 5 .

Step 4 Let

$$
\begin{aligned}
V_{i}= & \left\{w \in \Delta_{i} \mid-\left(\tilde{\boldsymbol{x}}^{(i-1)}\right)^{T} \tilde{\boldsymbol{C}}(w) \geq 0,\right. \\
& \left.\left(\tilde{\boldsymbol{x}}^{(i-1)}\right)^{T} \tilde{\boldsymbol{C}}(w)-\delta(w) \geq 0\right\} \cup E_{i-1} .
\end{aligned}
$$

Solve Problem $D P_{k}$ with $\boldsymbol{w}=V_{i}$ to obtain a solution $\left(\tilde{\boldsymbol{x}}^{(i)}, \boldsymbol{\mu}^{(i)}\right)$. Check the feasibility of $\tilde{\boldsymbol{x}}^{(i)}$ to Problem $\mathrm{P} 2$. If it is feasible, go to Step 8. Otherwise, set

$$
\begin{aligned}
E_{i}= & \left\{w \in \Delta_{i} \mid-\left(\tilde{\boldsymbol{x}}^{(i-1)}\right)^{T} \tilde{\boldsymbol{C}}(w)=0,\right. \\
& \left.\left(\tilde{\boldsymbol{x}}^{(i-1)}\right)^{T} \tilde{\boldsymbol{C}}(w)-\delta(w)=0\right\}
\end{aligned}
$$

and go to Step 3.

Step 5 Set $i=i+1$. Find $w_{i} \in \Delta_{i}$ such that

$$
\begin{gathered}
z_{i}=\arg \max _{w \in \Delta_{i}}\left\{-\left(\tilde{\boldsymbol{x}}^{(i-1)}\right)^{T} \tilde{\boldsymbol{C}}(w),\right. \\
\left.\left(\tilde{\boldsymbol{x}}^{(i-1)}\right)^{T} \tilde{\boldsymbol{C}}(w)-\delta(w)\right\} .
\end{gathered}
$$

If

$$
\max _{w \in \Delta_{i}}\left\{-\left(\tilde{\boldsymbol{x}}^{(i-1)}\right)^{T} \tilde{\boldsymbol{C}}(w),\left(\tilde{\boldsymbol{x}}^{(i-1)}\right)^{T} \tilde{\boldsymbol{C}}(w)-\delta(w)\right\}<\varepsilon
$$

set

$$
Z_{i}=E_{i-1}, \quad\left(\tilde{\boldsymbol{x}}(i), \boldsymbol{v}^{(i)}\right)=\left(\tilde{\boldsymbol{x}}^{(i-1)}, \boldsymbol{v}^{(i-1)}\right), E_{i}=E_{i-1},
$$

and repeat Step 5 .

Else, set

$$
Z_{i}=\left\{z_{i}\right\} \cup E_{i-1}, E_{i}=\left\{z_{i}\right\} \cup E_{i-1}
$$

End

Step 6 Solve Problem $D P_{k}$ with $\boldsymbol{w}=Z_{i}$ to obtain a solution $\left(\tilde{\boldsymbol{x}}^{(i)}, \boldsymbol{\mu}^{(i)}\right)$. Check the feasibility of $\tilde{\boldsymbol{x}}^{(i)}$ to Problem P2. If it is feasible, go to Step 8. Otherwise, go to Step 7.

Step 7 Choose a set $E_{i} \subset Z_{i}$ with no more than $\tilde{L}+2$ points such that the solution of Problem $D P_{i}$ is in the form of $\left(\tilde{\boldsymbol{x}}^{(i)}, \boldsymbol{\mu}^{(i)}\right)$. Go to Step 5 . 
Step 8 Find a local minimum $\left(\tilde{\boldsymbol{x}}^{*}, \boldsymbol{\mu}^{*}, \boldsymbol{w}^{*}\right)$ for Problem $D P_{k}$ starting from $\left(\tilde{\boldsymbol{x}}^{(i)}, \boldsymbol{\mu}^{(i)}, E_{i}\right)$. Then, $\tilde{\boldsymbol{x}}^{*}$ is taken as the solution for Problem P2.

Remark 2: Step 7 is achieved through solving the Phase 1 of a linear program using the simplex method. For details, see Lemma 3.1 in [14].

In Algorithm $1, \varepsilon>0$ is the tolerance. The set $Z_{i}$ is restricted to be a set with $\tilde{L}+2$ points through the implementation of Step 7. Thus, a semi-infinite optimization problem P2 is solved by solving a sequence of quadratic programming problems with $\tilde{L}$ linear equality constraints where their dimensions are not exceeding $3 \tilde{L}+10$. The current version of the dual parametrization method makes use of the superior features of the dual parametrization methods reported in [13] and [14]. The convergence of the algorithm can be proved similarly as that given for Theorem 3.1 in [14].

Now, Problem P1 can be solved by the following iterative scheme.

Algorithm 2:

Step 1 Set $\varepsilon$ to be a small number and $k=1$. Use Algorithm 1 to solve Problem P1 with $\lambda=0$ and denote the obtained solution as $\tilde{\boldsymbol{x}}^{(0)}$.

Step 2 Solve Problem P2 with $\tilde{\lambda}=\lambda /\left(\tilde{\boldsymbol{x}}^{(k-1)}\right)^{T} \tilde{\boldsymbol{x}}^{(k-1)}$ by Algorithm 1 and denote the obtained solution as $\tilde{\boldsymbol{x}}^{(k)}$. Go to Step 3.

Step 3 If $\left\|\tilde{\boldsymbol{x}}^{(k)}-\tilde{\boldsymbol{x}}^{(k-1)}\right\| \leq \varepsilon$, stop and $\tilde{\boldsymbol{x}}^{(k)}$ is regarded as the optimal solution. Otherwise, set $k=k+1$ and go to Step 2.

\section{NUMERICAL EXPERIMENTS}

Let us first consider the case for which no requirement is imposed on the robustness to timing jitter, i.e., $\lambda=0$ in (9). Since Problem P1 with $\lambda=0$ is a semi-infinite quadratic optimization problem with linear constraints, it can be solved by Algorithm 1. Let $N=109, M=5, \delta=2 \times 10^{-4}, \beta=0.25$. We use the discritization method in [15], which is used in [5], and our method to solve this problem. All the elements of the initial point $\tilde{\boldsymbol{x}}^{(0)}$ are set to be 1 . After 6 iterations, the optimal cost obtained by our method is $-170.6404 \mathrm{~dB}$ with only two active constraints. From (7), we know that the ratios of the tail energy to the total energy is $10 \log \left(E_{\text {tail }} / E_{\text {total }}\right)=-12.257 \mathrm{~dB}$. The simulations are implemented in a computer with Intel Core Duo CPU E6850 $3.00 \mathrm{GHz}$ and RAM 2 GB. The run time for our method and that for the method in [15] are, respective, 0.063324 seconds and 1.987125 seconds. The magnitudes and the zoom magnitudes in the stopband by our method and the method in [15] are depicted in Fig.1 and Fig. 2, respectively. From the obtained results, we observe that there is a significant improvement in the results obtained by our method over those obtained by the method in [15].

The trade-off between the stopband energy of the designed filter and its robustness to timing jitter is achieved by tuning $\lambda$. If $\lambda$ is large, then the designed filter will posses a good robustness to timing jitter but with a larger stopband energy. On the other hand, if $\lambda$ is small, the designed filter will have a small stopband energy but will be more sensitive to timing jitter. To see this, we use our method to design Nyquist filters with $\lambda=0.5$ and $\lambda=2$. In 
each case, their optimal results are obtained after 2 times of calling Algorithm 2. The ratios of their tail energy to the total energy are $-18.056 \mathrm{~dB}$ and $-37.909 \mathrm{~dB}$, respectively. The filter coefficients with $\lambda=0,0.5$ and $\lambda=2$, are depicted in Fig. 3 and Fig. 4 and their magnitudes are depicted in Fig. 5. The corresponding eye diagrams are given in Fig. 6, Fig. 7 and Fig. 8. From the results obtained, we see that as $\lambda$ is increased, the filter becomes more robustness to timing jitter, but with larger stopband energy. Therefore, by varying $\lambda$, the proposed method can balance the sensitivity to the timing jitter against the stopband attenuation.

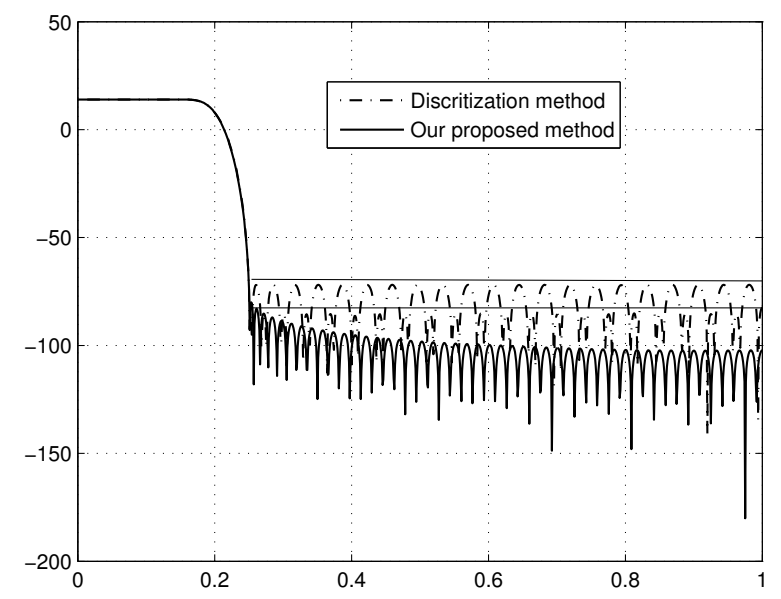

Fig. 1. The magnitudes response obtained by our method (solid line) and method in [15] (dot line)

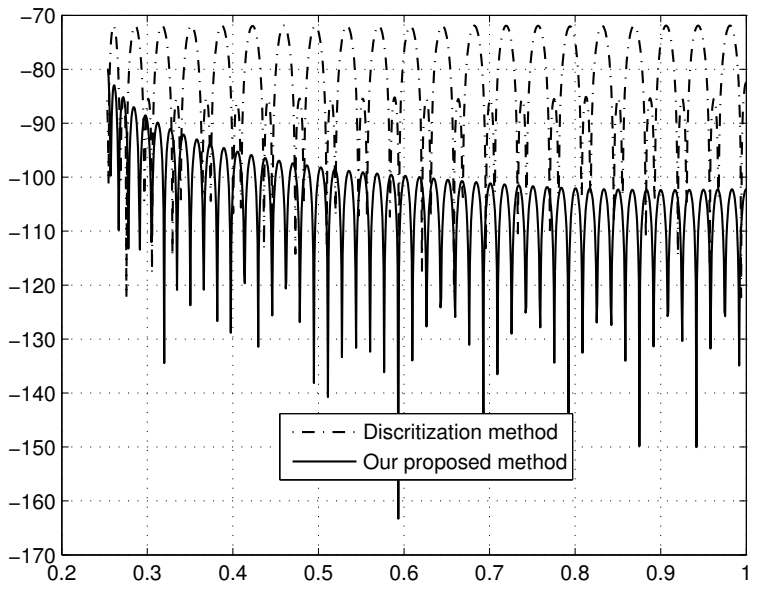

Fig. 2. The zoom magnitudes obtained by our method (solid line) and method in [15] (dot line)

Let $N=29, M=5, \beta=0.25$. First, the "rcosine" function in Matlab 7.1 is used to design the raised cosine function. The obtained ratio of the tail energy to the total energy is $-17.151 \mathrm{~dB}$ and the obtained stopband attenuation is more than $0.01 \mathrm{~dB}$. We set $\delta=0.01$ and use our method to design the filter with $\lambda=0.01$. The obtained ratio of the tail energy to the total energy is $-20.199 \mathrm{~dB}$. Thus, the filter obtained by our method is more robust to timing jitter than the raised cosine filter. All the results are depicted in Fig. 9 and Fig. 10. From Fig. 10 , we observe that the stopband attenuation has a significant improvement over that of the raised cosine function while achieving, at the same time, the improved robustness to timing jitter. 


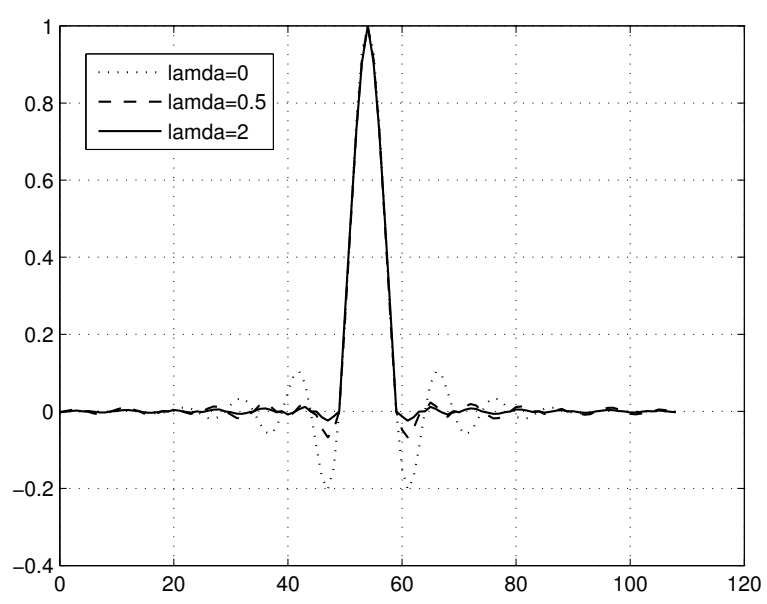

Fig. 3. The filter coefficients with $\lambda=0,0.5$, and $\lambda=2$

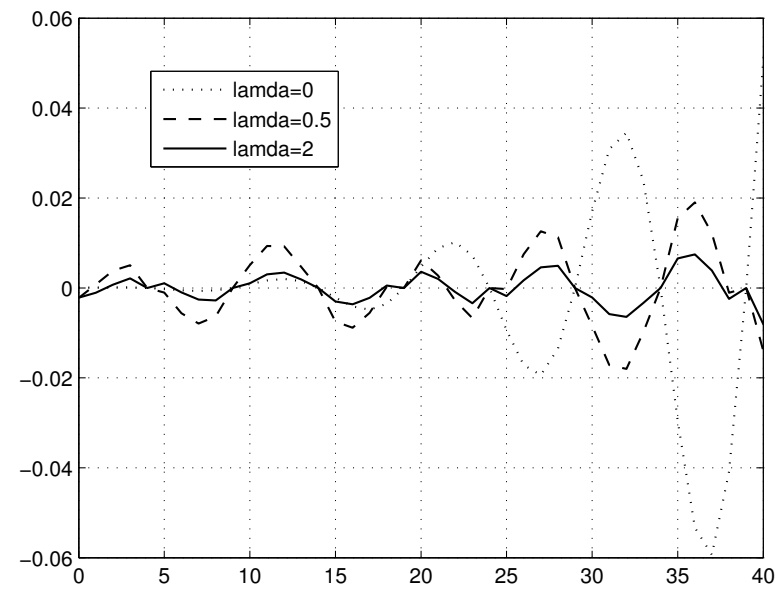

Fig. 4. The zoom filter coefficients with $\lambda=0,0.5$, and $\lambda=2$

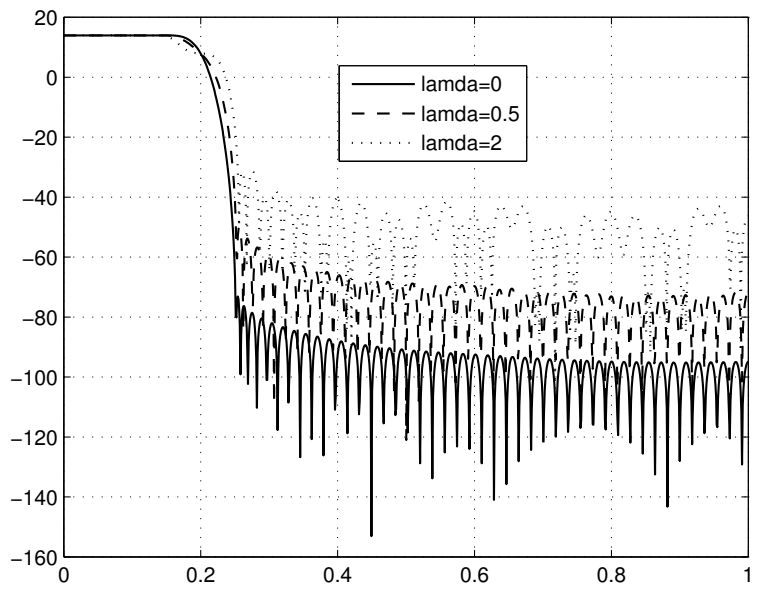

Fig. 5. The magnitude response with $\lambda=0,0.5$, and $\lambda=2$ (in $\mathrm{dB}$ ) 


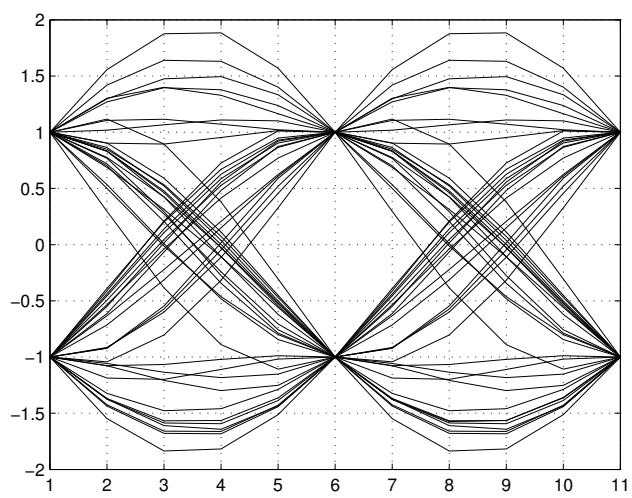

Fig. 6. The eye diagram with $\lambda=0$

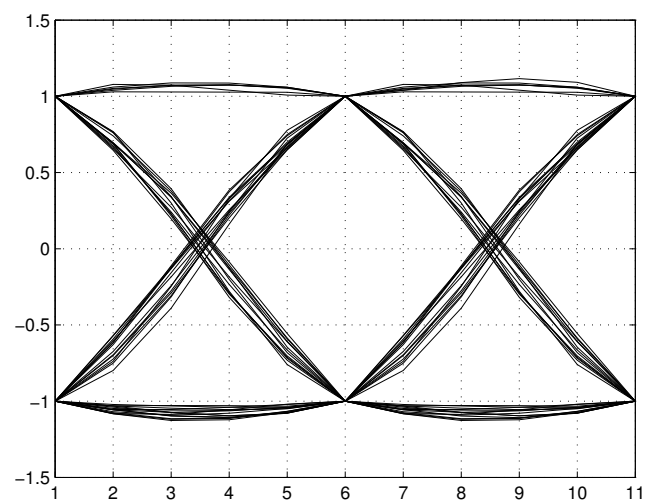

Fig. 7. The eye diagram with $\lambda=0.5$

\section{CONCLUSION}

In this paper, the design of a Nyquist filter satisfying the exact ISI condition, while maximizing the robustness to timing jitter is formulated as a nonlinear optimization problem with continuous inequality constraints. An efficient method combining an iterative scheme and dual parametrization techniques is developed. The trade-off between the robustness to timing jitter and the stopband attenuation is achieved by varying $\lambda$. The simulation results show that our method is very effective.

\section{REFERENCES}

[1] C.H. Muravchik and J.R. Guisantes, "Optimized signaling waveforms to reduce the effects of intersymbol interference and timing jitter”, IEEE Trans. Signal Process., Vol. 43, No. 1, 11-14, 1995.

[2] E.Panayiric, T. Ozugur and H. Caglar, "Design of optimum nyquist signals based on generalized sampling theory for data communcations”, IEEE Trans. Signal Process., Vol. 47, No. 6, 1753-1759, 1999.

[3] B.F. Boroujeny and G. Mathew, "Nyquist filters with robust performance against timing jitter", IEEE Trans. Signal Process., Vol. 46, No. 12, 3427-3431, 1998.

[4] B.F. Boroujeny, "A square-root Nyquist filter design for digital communications systems", IEEE Trans. Signal Process., Vol. 56, No. 5, 2127-2132, 2008.

[5] M. Liu, C.J. Zarowski and F.W. Fairman, "Factorable FIR Nyquist filters with least stopband energy under sidelobe level constraints", IEEE Trans. Signal Process., Vol. 48, No. 5, 1495-1498, 2000. 


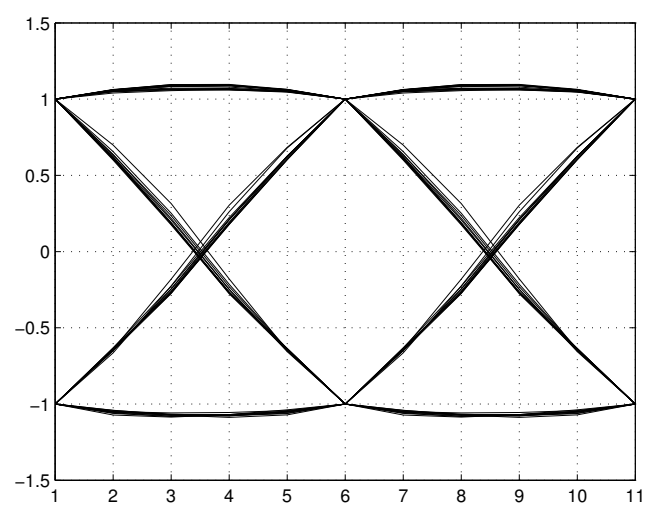

Fig. 8. The eye diagram with $\lambda=2$

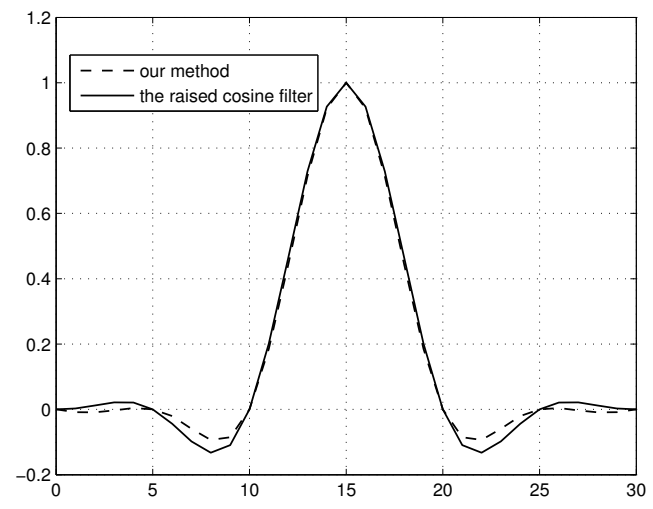

Fig. 9. The filter coefficients obtained by our method and those of the raised cosine filter

[6] X. Zhang and T. Yoshikawa, "Design of FIR Nyquist filters with low group delay", IEEE Trans. Signal Process., Vol. 47, No. 5, 1454-1458, 1999.

[7] T. Saramaki and Y. Neuvo, "A class of FIR Nyquist (Nth-band) filters with zeros intersymbol interference", IEEE Trans. Circuits Syst., Vol. 34, No. 10, 1182-1190, 1987.

[8] P. Vandamme, "On the synthesis of digital transmit filters", IEEE Trans. Commun., vol. 39, pp. 485-487, 1991.

[9] A.N.D. Andrea, F. Guglielmi, U. Mengali, and A. Spalvieri,, "Design of transmit and receive digital filters for data communications", IEEE Trans. Commun., vol. 42, pp. 357-359, 1994.

[10] T.N. Davidson, "Efficient design of waveforms for robust pulse amplitude modulation ”, IEEE Trans. Signal Process., Vol. 49, No. 12, 3098-3011, 2001.

[11] C. Z. Wu, K. L. Teo, V. Rehbock and H. H. Dam, "Global optimum design of uniform FIR filter Bank with magnitude constraints", IEEE Trans. Signal Process, Vol. 56, 5478-5486, 2008.

[12] K.L. Teo and X.Q. Yang, "A computational discretization algorithm for a functional inequity constrained optimization problem", Annals of Operations Research, Vol. 98, 215-234, 2000.

[13] Y. Liu and K.L. Teo, "An adaptive dual parameterization algorithm for quadratic semi-infinite programming problems", Journal of Global Optimization, Vol. 24, 205-217, 2002.

[14] Y. Liu, K.L. Teo and S.Y. Wu, "A new quadratic semi-infinite programming algorithm based on dual parameterization", Journal of Global Optimization, Vol. 29, 401-413, 2004.

[15] R. Hettich and K.O. Kortanek, "Semi-infinite programming: Theory, methods and applications", SIAM Rev., Vol. 35, No. 3, 380-429, 1993.

[16] Z.J. Zhang, P.L. Shui and T. Su, "Efficient design of high-complexity cosine modulated filter banks using $2 M$ th band conditions", 


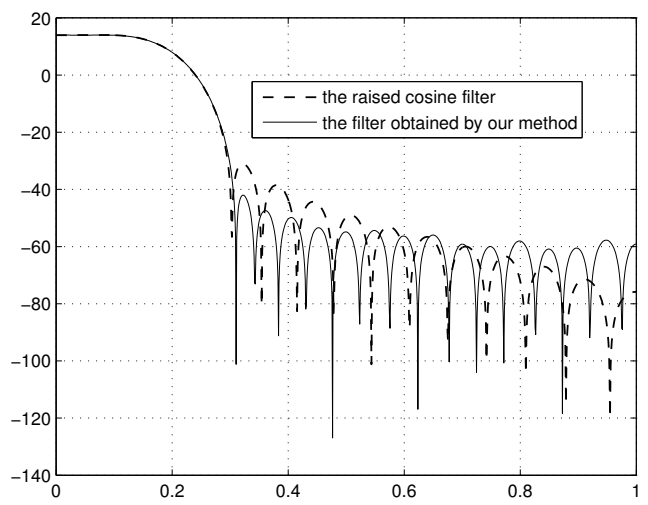

Fig. 10. The magnitude obtained by our method and that of the raised cosine filter (in $\mathrm{dB}$ )

IEEE Trans. Siggnal Process., Vol. 56, No. 11, 5414-5426, 2008. 